PROCEEDINGS OF THE

AMERICAN MATHEMATICAL SOCIETY

Volume 125, Number 5, May 1997, Pages 1299-1303

S 0002-9939(97)03748-9

\title{
A NOTE ON THE RELATIVE CLASS NUMBER IN FUNCTION FIELDS
}

\author{
MICHAEL ROSEN
}

(Communicated by William W. Adams)

\begin{abstract}
Let $F$ be a finite field, $A=F[T]$, and $k=F(T)$. Let $K_{m}=k\left(\Lambda_{m}\right)$ be the field extension of $k$ obtained by adjoining the $m$-torsion on the Carlitz module. The class number $h_{m}$ of $K_{m}$ can be written as a product $h_{m}=h_{m}^{+} h_{m}^{-}$. The number $h_{m}^{-}$is called the relative class number. In this paper a formula for $h_{m}^{-}$is derived which is the analogue of the Maillet determinant formula for the relative class number of the cyclotomic field of $p$-th roots of unity. Some consequences of this formula are also derived.
\end{abstract}

Let $\mathbf{Q}$ denote the rational numbers and consider the cyclotomic field $K_{p}=\mathbf{Q}\left(\zeta_{p}\right)$ with class number $h_{p}$. It is well known that this class numbers factors as a product $h_{p}^{+} h_{p}^{-}$of two integers. The number $h_{p}^{+}$is the class number of the maximal real subfield of $K_{p}$. The integer $h_{p}^{-}$is called the relative class number. In [Ca] and $[\mathrm{Ca}-\mathrm{O}]$ it is shown how the relative class number can be computed in terms of a certain classical determinant known as the Maillet determinant. A nice exposition of this is given in Chapter 3 of [L] (see Theorem 7.1).

In this note we will give an analogue of this material in the context of cyclotomic function fields. Let $F$ be a finite field with $q$ elements and $A=F[T]$ the polynomial ring over $F$. Let $k=F(T)$ be the quotient field of $A$. For an irreducible polynomial $m$ of degree $d$ we will denote by $\Lambda_{m}$ the $m$-torsion on the Carlitz module and let $K_{m}=k\left(\Lambda_{m}\right)$ be the "cyclotomic" function field obtained by adjoining the elements of $\Lambda_{m}$ to $k$. For the definition of the Carlitz module and its properties see $[\mathrm{H}]$ and [G-R]. The class number of $K_{m}, h_{m}$, factors as a product of two integers $h_{m}=h_{m}^{+} h_{m}^{-}$, where $h_{m}^{+}$is the class number of the "maximal real" subfield of $K_{m}$, i.e. the decomposition field of the prime at infinity of $k$ in $K_{m}$, and $h_{m}^{-}$is called the relative class number. Our aim is to give an expression for $h_{m}^{-}$as a product of certain easily computed determinants related to the classical Maillet determinant.

We begin by recalling the analytic class number formula for $h_{m}^{-}$which follows immediately from Theorem 2 of [G-R]. A character $\chi$ of $(A / m A)^{*}$ is said to be real if its restriction to $F^{*}$ is the trivial character. Otherwise it is said to be non-real or imaginary. If $\chi$ is imaginary, define $S(\chi)=\sum_{a} \chi(a)$, where the sum is over all monic polynomials of degree less than $d$. Then,

$$
h_{m}^{-}=\prod_{\chi \text { imaginary }} S(\chi) .
$$

Received by the editors July 2, 1995 and, in revised form, November 15, 1995.

1991 Mathematics Subject Classification. Primary 11R29; Secondary 11R58, 14H05.

This work was partially supported with a grant from the National Science Foundation. 
Let $t=\left(q^{d}-1\right) /(q-1)$. Then $t$ is the size of the set $\mathcal{M}$ of monic polynomials of degree less than $d$. We construct a $t \times t$ matrix $[c(a, b)]$ where $a, b \in \mathcal{M}$. Namely, for $a, b \in \mathcal{M}$ write $a b=s m+r$, where $s, r \in A$ and either $r=0$ or $\operatorname{deg}(r)<d$. In fact, $r$ cannot be zero since we have assumed that $m$ is irreducible. Define $c(a, b)$ to be the leading coefficient of $r$. By construction, the matrix $[c(a, b)]$ has all its coefficients in $F^{*}$. Let $\lambda$ be a character of $F^{*}$ and define

$$
d(\lambda)=\operatorname{det}[\lambda(c(a, b))]
$$

Theorem 1.

$$
h_{m}^{-}= \pm \prod_{\lambda \neq \lambda_{o}} d(\lambda)
$$

where the product is over all the non-trivial characters $\lambda$ of $F^{*}$.

Proof. The proof proceeds by a step by step rewriting of equation (1). Let $\lambda$ be any non-trivial character of $F^{*}$, and set

$$
h_{\lambda}=\prod_{\left.\chi\right|_{F^{*}=\lambda}} S(\chi)
$$

From equation (1) we see that

$$
h_{m}^{-}=\prod_{\lambda \neq \lambda_{o}} h_{\lambda}
$$

Let $\phi$ be a fixed character whose restriction to $F^{*}$ is $\lambda$. Then all the characters whose restriction to $F^{*}$ is $\lambda$ can be written $\phi \chi^{\prime}$, where $\chi^{\prime}$ is a real character. Note that a real character can also be thought of as a character of the group $G=(A / m A)^{*} / F^{*}$. We'll come back to this in a moment.

Starting with a non-trivial character $\lambda$ on $F^{*}$ we construct a function $\tilde{\lambda}$ on $(A / m A)^{*}$ as follows. Let $a$ be a polynomial prime to $m$. Write $a=s m+r$ with $s, r \in A$ and $\operatorname{deg}(r)<d$. Define $\tilde{\lambda}(a)$ to be $\lambda$ evaluated on the inverse of the leading coefficient of $r$. Then $\tilde{\lambda}$ is a well defined function on $(A / m A)^{*}$.

We rewrite $h_{\lambda}$ using the quantities just defined:

$$
\begin{gathered}
h_{\lambda}=\prod_{\left.\chi\right|_{F^{*}=\lambda}} \sum_{a \text { monic, } \operatorname{deg}(a)<d} \chi(a) \\
=\prod_{\chi^{\prime}} \sum_{a \text { monic, } \operatorname{deg}(a)<d} \chi^{\prime}(a) \phi(a) \tilde{\lambda}(a) .
\end{gathered}
$$

Observe that the function $\phi(a) \tilde{\lambda}(a)$ is unchanged if we replace $a$ by $\alpha a$ where $\alpha$ is a constant. It is also unchanged if we replace $a$ by anything in the congruence class of $a$ modulo $m$. Thus the summation in the above sum can be rewritten as a summation over all the elements of the group $G=(A / m A)^{*} / F^{*}$. We obtain

$$
h_{\lambda}=\prod_{\chi^{\prime}} \sum_{a \in G} \chi^{\prime}(a)[\phi(a) \tilde{\lambda}(a)] .
$$

We apply the Dedekind determinant formula (see [L], Theorem 6.1) to derive

$$
h_{\lambda}=\operatorname{det}\left[\phi\left(b^{-1} a\right) \tilde{\lambda}\left(b^{-1} a\right)\right] \text {. }
$$


Here, $a$ and $b$ vary over the elements of the group $G$. Replacing $b^{-1}$ by $b$ merely permutes the rows of the matrix, and so,

$$
h_{\lambda}= \pm \operatorname{det}[\phi(b) \phi(a) \tilde{\lambda}(b a)] .
$$

We have used the fact that $\phi$ is a character. Each element of the $b^{\prime}$ th row of the determinant in equation (3) contains $\phi(b)$ as a factor. Similarly, each element of the $a^{\prime}$ th column contains $\phi(a)$ as a factor. By elementary properties of determinants, it follows that

$$
h_{\lambda}= \pm \phi\left(\prod_{a \in G} a\right)^{2} \operatorname{det}[\tilde{\lambda}(a b)] .
$$

The product of all the elements in an abelian group is equal to the product of all the elements of order two. Thus,

$$
\phi\left(\prod_{a \in G} a\right)^{2}=( \pm 1)^{2}=1
$$

and so,

$$
h_{\lambda}= \pm \operatorname{det}[\tilde{\lambda}(a b)]= \pm d\left(\lambda^{-1}\right) .
$$

The last equality is obtained by letting $a$ and $b$ once again run through all the monic polynomials of degree less than $d$ and simply using the definition of $\tilde{\lambda}$. From equations (2) and (4) the proof of the theorem is immediate.

I would like to thank David Goss for suggesting that the following result should hold.

Theorem 2. Let $[c(a, b)]$ be the matrix introduced in the remarks preceding Theorem 1. Then, the relative class number $h_{m}^{-}$is divisible by $p$, the characteristic of $F$, if and only if there is an integer $i, 1 \leq i \leq q-2$, such that $\operatorname{det}\left[c(a, b)^{i}\right]=0$.

Proof. We have to reinterpret equation (2) first $p$-adically and then, after reduction $\bmod p$, as an equality in the ring $A / m A$.

Let $\zeta$ be a complex primitive $\left(q^{d}-1\right)$ st root of unity and set $E=\mathbf{Q}(\zeta)$. Let $\mathcal{O}$ be the ring of integers in $E$. $E$ is unramified at all primes above $p$. Let $\mathcal{P}$ be a fixed such prime and let $\hat{\mathcal{O}}$ be the completion of $\mathcal{O}$ at $\mathcal{P}$. Using the natural imbedding of $\mathcal{O}$ in $\hat{\mathcal{O}}$ we can (and do) interpret equation (2) as holding in $\hat{\mathcal{O}}$. Now, the residue class field, $\kappa$, of $\hat{\mathcal{O}}$ is a finite field with $q^{d}$ elements and so is isomorphic to $A / m A$. Let $\rho: \kappa \rightarrow A / m A$ be such an isomorphism. Finally, let $r$ denote reduction modulo $\hat{\mathcal{P}}$ on $\hat{\mathcal{O}}$. Applying the composed map $\rho \circ r$ to both sides of equation (2), we see that $p$ divides $h_{m}^{-}$if and only if $\rho \circ r\left(h_{\lambda}\right)=0$ in $A / m A$ for some complex character $\lambda$ of $F^{*}$.

Let $\lambda$ be any complex character of $F^{*}$, and define $\tilde{\lambda}=\rho \circ r \circ \lambda$. Then $\tilde{\lambda}$ is a character on $F^{*}$ with values in the $(q-1)$ st roots of unity in $(A / m A)^{*}$, i.e. in $F^{*}$. It is easy to see that the multiplicative maps from $F^{*}$ to itself consist of precisely the powers of the identity map under pointwise product. Now, apply the homomorphism $\rho \circ r$ to both sides of equation (4). We find

$$
\rho \circ r\left(h_{\lambda}\right)= \pm d\left(\rho \circ r \circ \lambda^{-1}\right)= \pm \operatorname{det}\left(c(a, b)^{i}\right),
$$

where $i$ is some index between 1 and $q-2$. This proves the theorem. 
Remark 1. It is undoubtedly true that Theorem 2 can be refined so that the vanishing of $\operatorname{det}\left[c(a, b)^{i}\right]$ can be related to the $p$-divisibility of the order of the "ith piece" of the class group. See [G-S] for an explanation of how to decompose the class group into pieces corresponding to powers of the Teichmüller character. We shall not enter into this here.

As in $[\mathrm{Ca}],[\mathrm{Ca}-\mathrm{O}]$, and $[\mathrm{L}]$, it is possible to use Theorem 1 to give an upper bound for $h_{m}^{-}$.

Theorem 3. As above, let $m \in A$ be an irreducible polynomial of degree $d$ and define $t=q^{d}-1 /(q-1)$. We have

$$
h_{m}^{-} \leq \sqrt{t}^{t(q-2)}<(2 q)^{\frac{d-1}{2} q^{d}} .
$$

Proof. We apply the Hadamard determinant inequality which states that the absolute value of the determinant of a complex square matrix is less than or equal to the product of the lengths of the row vectors which compose the matrix. Since all the entries of $[\lambda(c(a, b))]$ have absolute value 1 , we find that $|d(\lambda)| \leq \sqrt{t}^{t}$. The first inequality follows from this and equation (2). As for the second inequality, note that the exponent $t(q-2)<q^{d}-1<q^{d}$. Also, note that $t=q^{d-1}+q^{d-2}+\ldots+1<2 q^{d-1}$. These two observation yield the second inequality.

It is interesting to compare the inequalities of Theorem 3 with those obtained from the congruence Riemann hypothesis. Recall that $h_{m}^{-}=h_{m} / h_{m}^{+}$. It follows from the theory of algebraic curves over finite fields and the congruence Riemann hypothesis that there are complex numbers $\pi_{i}$ of absolute value $\sqrt{q}$ such that

$$
h_{m}^{-}=\prod_{i=1}^{2 g-2 g^{+}}\left(1-\pi_{i}\right) .
$$

Here, $g$, resp. $g^{+}$, is the genus of the field $K_{m}$, resp. $K_{m}^{+}$. The only primes of $k$ which ramify in $K_{m}$ are $(m)$ and $\infty$. The prime $(m)$ is totally ramified of degree $d$, whereas $\infty$ splits into $t$ primes each with ramification index $q-1$ and degree 1 . By Riemann-Hurwitz one deduces that

$$
2 g-2=(d-1) q^{d}+1-2 d-t .
$$

See $[\mathrm{H}]$ for more details and the case of general $m$. In $K_{m}^{+}$only $(m)$ is ramified, and it is totally ramified. It follows that

$$
2 g^{+}-2=(d-2) t-d .
$$

Subtracting these equations, we find that

$$
2 g-2 g^{+}=(d-1) q^{d}+O\left(q^{d-1}\right) .
$$

The error term is an explicit polynomial in $q$ whose leading term is $(1-d) q^{d-1}$, so for large $d$ the error term makes a negative contribution.

Combining equations (6) and (7) and using the very coarse inequality $\sqrt{q}+1<$ $2 \sqrt{q}$, we deduce that

$$
h_{m}^{-}<(\sqrt{q}+1)^{2 g-2 g^{+}}<(4 q)^{g-g^{+}} .
$$

Equations (7) and (8) yield

$$
\log _{q}\left(h_{m}^{-}\right)<\left(1+\frac{\log (4)}{\log q}\right) \frac{d-1}{2} q^{d}+O\left(q^{d-1}\right),
$$


whereas the elementary methods used in proving Theorem 3 (see equation (5)) give the result

$$
\log _{q}\left(h_{m}^{-}\right)<\left(1+\frac{\log (2)}{\log (q)}\right) \frac{d-1}{2} q^{d},
$$

which is surprisingly good.

We conclude with two more remarks.

Remark 2. Throughout this paper we have been concerned with the class numbers associated to the fields $K_{m}$ and $K_{m}^{+}$. It is also of interest to consider the class numbers of the rings $\mathcal{O}_{m}$ and $\mathcal{O}_{m}^{+}$which are the integral closures of $A$ in $K_{m}$ and $K_{m}^{+}$respectively. Call these class numbers $h\left(\mathcal{O}_{m}\right)$ and $h\left(\mathcal{O}_{m}^{+}\right)$. It can be shown that the second of these numbers divides the first. Call the ratio $h\left(\mathcal{O}_{m}\right)^{-}$. This is the relative class number on the level of rings. The following relationship holds:

$$
h\left(\mathcal{O}_{m}\right)^{-}=(q-1)^{1-t} h_{m}^{-} \text {. }
$$

For a proof of this, in a more general setting, see $[R]$.

The upshot is that Theorem 1 provides a formula for $h\left(\mathcal{O}_{m}\right)^{-}$as well as $h_{m}^{-}$. Also, Theorem 2 gives a criterion for $p$-divisiblity of both of these numbers.

Remark 3. The "cyclotomic" construction of ray class fields of $k=F(T)$ due to Carlitz has been extended to arbitrary global function fields by V.G. Drinfeld and D.R. Hayes. Using Hayes' normalized rank one Drinfeld modules, L. Shu [S] has developed analytic class number formulas for the generalization of the relative class numbers $h_{m}^{-}$. Using these, she is has been able to extend Theorems 1 and 2 to this setting. Her paper is in preparation.

\section{REFERENCES}

[Ca] L. Carlitz, A generalization of Maillet's determinant and a bound for the first factor of the class number, Proc. AMS 12 (1961), 256-261. MR 22:12093

[Ca-O] L. Carlitz and F.R. Olson, Maillet's Determinant, Proc. AMS 6 (1955), 265-269. MR 16:999d

[G-R] S. Galovich and M. Rosen, The Class Number of Cyclotomic Function Fields, J. of Number Theory 13, No. 3 (1981), 363-375. MR 83m:12022

[G-S] D. Goss and W. Sinnott, Class-groups of Function Fields, Duke Math. J. 52, No. 2 (1985), 507-516. MR 87b:11118

$[\mathrm{H}] \quad$ D. Hayes, Explicit class field theory for rational function fields, Trans. Amer. Math. Soc. 189 (1974), 77-91. MR 48:8444

[L] S. Lang, Cyclotomic Fields, Springer Verlag, New York-Heidelberg-Berlin, GTM 59, 1978. MR 58:5578

[R] M. Rosen, The Hilbert class field in function fields, Exposition. Math. 5 (1987), 365-378. MR 89b:10094

[S] L. Shu, Narrow ray class fields and partial zeta functions, pre-print, 1995.

Department of Mathematics, Brown University, Providence, Rhode Island 02912-0001

E-mail address: ma408000@brownvm.brown.edu 